\title{
Words with many palindrome pair factors
}

\author{
Adam Borchert and Narad Rampersad * \\ Department of Mathematics and Statistics \\ University of Winnipeg \\ 515 Portage Avenue \\ Winnipeg, Manitoba R3B 2E9, Canada \\ adamdborchert@gmail.com, n.rampersad@uwinnipeg.ca \\ Submitted: Sep 19, 2015; Accepted: Oct, 16, 2015; Published: Oct 30, 2015 \\ Mathematics Subject Classifications: 68R15
}

\begin{abstract}
Motivated by a conjecture of Frid, Puzynina, and Zamboni, we investigate infinite words with the property that for infinitely many $n$, every length- $n$ factor is a product of two palindromes. We show that every Sturmian word has this property, but this does not characterize the class of Sturmian words. We also show that the Thue-Morse word does not have this property. We investigate finite words with the maximal number of distinct palindrome pair factors and characterize the binary words that are not palindrome pairs but have the property that every proper factor is a palindrome pair.
\end{abstract}

\section{Introduction}

The palindromic length of a word $x$ is the least $\ell$ such that $x=p_{1} p_{2} \cdots p_{\ell}$, where each $p_{i}$ is a palindrome (we consider the empty word a palindrome). Frid, Puzynina, and Zamboni [6] made the following, remarkable conjecture:

Conjecture (Frid-Puzynina-Zamboni). Let $\mathbf{w}$ be an infinite word. If there exists $k$ such that every factor of $w$ has palindromic length at most $k$, then $\mathbf{w}$ is ultimately periodic.

In this paper we focus on words with palindromic length at most 2: these are called palindrome pairs and have been studied before [9, 10, 11]. Assuming that the FridPuzynina-Zamboni Conjecture is true, there is no aperiodic word with the property that all of its factors are palindrome pairs. We therefore investigate the following question, with the hope that it will give some insight into the conjecture.

*The first author is supported by an NSERC USRA, the second by an NSERC Discovery Grant. 
Are there aperiodic words $\mathbf{w}$ with the property $(\mathbf{P P})$ that for infinitely many $n$, every length- $n$ factor of $\mathbf{w}$ is a palindrome pair?

As we will see, all Sturmian words have property PP, but this does not characterize the Sturmian words: there are other words with this property as well. We also show that the Thue-Morse word does not have property PP. We then look at the analogue of (finite) rich words for palindrome pairs, rather than palindromes. Finally, we characterize the finite binary words that are not palindrome pairs, but have the property that each of their proper factors is a palindrome pair.

\section{Palindrome pairs in Sturmian words}

In this section we show that every Sturmian word $\mathbf{s}$ has property $\mathbf{P P}$ and we characterize the lengths $n$ for which every length- $n$ factor of $\mathbf{s}$ is a palindrome pair. We begin with some preliminaries concerning Sturmian words.

Let $\alpha$ be an irrational real number (fixed for the remainder of this section) with $0<\alpha<1$ and let $\alpha$ have the continued fraction expansion $\alpha=\left[0 ; a_{1}+1, a_{2}, a_{3}, \ldots\right]$. We define the following sequence of words:

$$
s_{0}=1, \quad s_{1}=0, \quad s_{n}=s_{n-1}^{a_{n-1}} s_{n-2} \text { for } n \geqslant 2 .
$$

Since $s_{n}$ is a prefix of $s_{n+1}$ for all $n \geqslant 1$, this sequence converges to an infinite word $\mathbf{c}_{\alpha}$ called the characteristic (or standard) Sturmian word with slope $\alpha$. An infinite word $\mathbf{s}$ is a Sturmian word with slope $\alpha$ if it has the same set of factors as $\mathbf{c}_{\alpha}$. Since we are only concerned with the language of factors of $\mathbf{s}$, we will assume without loss of generality that $\mathbf{s}=\mathbf{c}_{\alpha}$. There are many equivalent definitions of "Sturmian word". For instance, another definition is the following: An infinite word is Sturmian if it has $n+1$ factors of length $n$ for every $n \geqslant 0$. For more details on Sturmian words see [12, Chapter 2].

Recall that words $x$ and $y$ are conjugates if $x=u v$ and $y=v u$ for some words $u$ and $v$.

Lemma 1. Any conjugate of a palindrome pair is a palindrome pair.

Proof. Let $A_{0} B_{0}$ be a palindrome pair where $A_{0}=a_{1} a_{2} \cdots a_{r}$ and $B_{0}=b_{1} b_{2} \cdots b_{s}$ are palindromes. As the result is trivial for the empty word, we may assume without loss of generality that $r \geqslant 1$ and $s \geqslant 0$. If $r=1$, note that $B_{0} a_{1}$ is a palindrome pair. Assume that $r \geqslant 2$. Then, since $A_{1}=a_{2} a_{3} \cdots a_{r-1}$ and $B_{1}=a_{r} b_{1} b_{2} \cdots b_{s} a_{1}$ are palindromes it follows that $A_{1} B_{1}$ is a palindrome pair. The result follows on repeated application of this argument.

Lemma 2. [4] A palindrome $p$ is a palindromic prefix of $\mathbf{s}$ if and only if one of the following hold:

- $p$ is a factor of $s_{1}^{a_{1}}$. 
- For either $u=p 01$ or $u=p 10$ we have $u=s_{n-1}^{k} s_{n-2}$ for some $n \geqslant 2$ and some $k$ such that $1 \leqslant k \leqslant a_{n-1}$.

Lemma 3. [3] For every $n \geqslant 2$ and every $k$ such that $1 \leqslant k \leqslant a_{n-1}, s_{n-1}^{k} s_{n-2}$ is a palindrome pair.

If $w$ is a word (finite or infinite), the notation $w[i: j]$ indicates the factor of $w$ beginning at position $i$ and ending at position $j-1$ in $w$. Unless otherwise stated, we assume that positions are indexed starting with 0 .

Lemma 4. Let $w$ be a left special factor of $\mathbf{s}$ such that neither $w[0:|w|-1]$ nor $w$ is a palindrome. The words $1 w$ and $0 w$ are not both palindrome pairs.

Proof. Let $w$ be such a factor. Since $w$ is left special, it is well known that $w$ occurs as a prefix of s. Assume without loss of generality that $1 w$ is a palindrome pair. Write $1 w=A B$ for palindromes $A$ and $B$. Based on our hypotheses on $w$, it follows from Lemma 2 that $|A| \neq 0,1$. Hence, we may write $A=1 P_{1} 1$ for a palindrome $P_{1}$. Since $P_{1}$ is a palindromic prefix of $\mathbf{s}$ it then follows from Lemma 2 that $B$ has prefix 0. Then, since $B$ is a palindrome it follows that $B$ (and hence, $w$ ) has suffix 0 . If $0 w$ were a palindrome pair it would follow by a similar argument that $w=0 p_{2} 01 \ldots 1$ and thus $w$ has suffix 1 , which is impossible.

Lemma 5. Let $u=s_{n-1}^{k} s_{n-2}$ for some $n \geqslant 4$ and some $k$ such that $1 \leqslant k \leqslant a_{n-1}$, where $|u| \geqslant 3 a_{1}+6$. There is a factor of $\mathbf{s}$ of length $|u|-1$ which is not a palindrome pair.

Proof. Let $a=0^{a_{1}}$ and $b=0^{a_{1}+1}$. Note that these are the only two maximal blocks of zeros occurring in $\mathbf{s}$. Let $w$ be the right special factor of $\mathbf{s}$ of length $|u|-2$. Note that since $w 0$ is a factor of $\mathbf{s}$, and since $b$ is maximal, that $w$ cannot have suffix $b$. Thus $w$ is a palindromic prefix of $\mathbf{s}$ with suffix (and hence, prefix) $a$. We consider the word $v=0^{-1} w 01$. Note that $|v|=|u|-1$. It follows from the facts that $w$ is right special and that $b$ is the maximal block of zeros in $\mathbf{s}$ that $v$ occurs in $\mathbf{s}$. If $v$ is not a palindrome pair we are done. Assume otherwise, and write $v=P_{1} P_{2}$ for palindromes $P_{1}$ and $P_{2}$. Suppose that $P_{1} \neq 0^{-1} a$. It then follows that

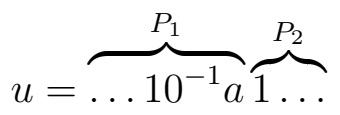

which contradicts that $a$ is the shortest maximal block of zeros in $\mathbf{s}$. Hence, $P_{1}=0^{-1} a$.

Thus we have $w 01=a P_{2}=a 1 \ldots 1 b 1$. Since $P_{2}$ is a palindrome it follows that $P_{2}=1 b 1 \ldots 1 b 1$. Then, since $w$ is a palindrome it follows that $w 01=a P_{2}=a 1 b 1 \ldots 1 b 1 b 1$. Continuing in this manner we obtain that $P_{2}=1(b 1)^{k}$ for some integer $k \geqslant 0$. We now consider two cases depending on which of $w 0$ or $w 1$ occurs as a prefix of $\mathbf{s}$.

Case 1. Assume that $w 0$ is a prefix of $\mathbf{s}$. Observe that $v$ occurs as a suffix of $s_{n}$, which itself is a prefix of $\mathbf{s}$. Let $\mathbf{s}[i: j]$ be this occurrence of $v$. Since the prefix $s_{n}$ is either followed by $s_{n-1}$, or $s_{n}$ (which has prefix $s_{n-1}$ ) and since $n \geqslant 4$, we then have either 


$$
\mathbf{s}=\overbrace{a 1 b 1 b \ldots \underbrace{}_{\mathbf{s}[i: j]} \ldots b 1}^{s_{n}} \overbrace{a 1 b \ldots . .}^{s_{n}-1} \ldots
$$

or

$$
\mathbf{s}=\overbrace{a 1 b 1 b \ldots \underbrace{s_{4}}_{\mathbf{s}[i: j]}}^{s_{4}} \overbrace{a 10}^{s_{3}} \overbrace{a 1}^{s_{2}} \ldots
$$

depending on the length of $s_{n-1}$.

Now, let $x=\mathbf{s}[i+2|b|: j+2|b|]$. Note that $|x|=|v|=|u|-1$. Suppose for contradiction that $x$ is a palindrome pair. Note that $x$ has prefix $b$ and suffix $1 a 1 b$ where $1 a 1$ is unioccurrent in $x$. Since $x$ is a palindrome pair it then follows that $x$ is a palindrome, and hence that $x=b 1 a 1 b$. Thus, $|u|=3|b|+2<3 a_{1}+6$, contrary to our hypothesis.

Case 2. We now assume that $w 1$ is a prefix of s. Note that $w 1=a 1(b 1)^{k} a 1$ for some $k>0$, and that $w 10=u$. As before, $u$ is either followed by $s_{n-1}$, or $s_{n}$ (which has prefix $\left.s_{n-1}\right)$. Thus,

$$
\mathbf{s}=\overbrace{w}^{\overbrace{a 1 b 1 b \ldots b 1 a}^{u} 10} \overbrace{a 1 \ldots .}^{s_{n}-1} \ldots
$$

Set $x=\mathbf{s}[|b|:|u|+|a|]$. Again, note that $|x|=|u|-1$. We suppose that $x$ is a palindrome pair and apply the same argument to $x$ as was done in the previous case to obtain $x=b 1 a 1 b$ and thus that $|u|<3 a_{1}+6$, a contradiction. This completes the proof.

Lemma 6. If $n \neq\left|s_{m-1}^{k} s_{m-2}\right|$ for any $m \geqslant 2$ and any $k$ such that $1 \leqslant k \leqslant a_{m-1}$, and $n \geqslant 3 a_{1}+6$, then $\mathbf{s}$ has at most $n-1$ palindrome pair factors of length $n$.

Proof. This follows from Lemmas 4 and 5, the fact that $\mathbf{s}$ has $n+1$ factors of length $n$, and the fact that the factor set of $\mathbf{s}$ is closed under reversal.

Theorem 7. Let $\mathbf{s}$ be a Sturmian word and let $n \geqslant 3 a_{1}+6$ be a positive integer. Every factor of $\mathbf{s}$ of length $n$ is a palindrome pair if and only if $n=\left|s_{m-1}^{k} s_{m-2}\right|$ for some $m \geqslant 4$ and some $k$ such that $1 \leqslant k \leqslant a_{m-1}$.

Proof. Let $n$ be such an integer. It is known that the $n+1$ factors of length $n$ in $\mathbf{s}$ are the conjugates of $s_{m-1}^{k} s_{m-2}$, as well as a palindromic singular factor [13]. It follows from Lemma 3 that $s_{m-1}^{k} s_{m-2}$ is a palindrome pair. It then follows from Lemma 1 that all factors of length $n$ are palindrome pairs. The converse follows immediately from Lemma 6.

Corollary 8. Every Sturmian word has property PP. 
Property PP does not characterize Sturmian words. Consider the following construction. For $i=1,2, \ldots$ let $w_{i}$ be an arbitrary palindrome of some length $t_{i}$ over $\{0,1\}$ and write $w_{i}=w_{i, 1} w_{i, 2} \cdots w_{i, t_{i}}$. We define a sequence of palindromes $p_{1}, p_{2}, \ldots$ as follows:

$$
\begin{aligned}
& p_{1}=0 \\
& p_{2}=p_{1} w_{1,1} p_{1} w_{1,2} \cdots p_{1} w_{1, t_{1}} p_{1} \\
& p_{3}=p_{2} w_{2,1} p_{2} w_{2,2} \cdots p_{2} w_{2, t_{2}} p_{2}
\end{aligned}
$$

Note that $p_{i}$ is a prefix of $p_{i+1}$ for all $i$, so we have a limiting infinite word $\mathbf{p}$.

Proposition 9. For each $i \geqslant 1$, every factor of $\mathbf{p}$ of length $\left|p_{i}\right|+1$ is a palindrome pair.

Proof. Any factor of $\mathbf{p}$ of length $\left|p_{i}\right|+1$ is a conjugate of $p_{i} a$ for some $a \in\{0,1\}$. The result follows from Lemma 1.

Now the word $\mathbf{p}$ is not necessarily Sturmian. For a suitable choice of $w_{i}$, we have that $0 p_{i} 0$ and $1 p_{i} 1$ are both factors of $\mathbf{p}$. However it is well-known that this implies that $\mathbf{p}$ is not Sturmian (see [12, Chapter 2]).

\section{Palindrome pairs in the Thue-Morse word}

Let $\mu$ be the morphism that maps $0 \rightarrow 01$ and $1 \rightarrow 10$. Let $\mathbf{t}=\mu^{\omega}(0)$ be the Thue-Morse word.

Theorem 10. The word $\mathbf{t}$ does not have property $\mathbf{P P}$.

Proof. This can be verified "automatically" using the Walnut Prover software created by Hamoon Mousavi and available at

$$
\text { https://cs. uwaterloo.ca/ shallit/papers.html . }
$$

We will not explain in detail the methodology implemented by the Walnut Prover (the reader may consult [8] or [14], for example). The important point is that to be able to apply this method, we need the property $\mathbf{P P}$ to be expressible in a certain extension of first order logic. The relevant formulae are given below. The first defines all pairs $(i, j)$ such that $\mathbf{t}[i: j]$ is a palindrome:

$$
(i, j):(i=j) \vee((i<j) \wedge \forall k(i+k<j) \Rightarrow \mathbf{t}[i+k]=\mathbf{t}[j-1-k]) .
$$

Let us refer to this formula as palindrome $(i, j)$. The clause $(i=j)$ corresponds to the empty palindrome when used in the next formula, which defines all $n$ such that every factor of $\mathbf{t}$ is a palindrome pair:

$$
(n): \forall i \exists j \text { palindrome }(i, j) \wedge \text { palindrome }(j, i+n) .
$$


The output of the Walnut Prover is a description (by a finite automaton) of the binary representations of all $n$ defined by the formula above. After running the prover we find that for $0 \leqslant n \leqslant 5$, every length- $n$ factor of $\mathbf{t}$ is a palindrome pair, but for $n \geqslant 6$ there is at least one length- $n$ factor of $\mathbf{t}$ that is not a palindrome pair.

Proposition 11. For each $k \geqslant 1$ there are at least $3 \cdot 2^{2 k+1}$ factors of $\mathbf{t}$ of length $3 \cdot 2^{2 k}$ that are palindrome pairs.

Proof. It is well known that for each $k$ the squares $\mu^{k}(010) \mu^{k}(010)$ and $\mu^{k}(101) \mu^{k}(101)$ occur in the Thue-Morse word. Furthermore, since $\mu^{2}(0)=0110$ and $\mu^{2}(1)=1001$, we see that $\mu^{2 k}(010)$ and $\mu^{2 k}(101)$ are palindromes. Thus every conjugate of $\mu^{2 k}(010)$ and $\mu^{2 k}(101)$ is a product of two palindromes and occurs in $\mathbf{t}$.

Proposition 12. For odd $n$ there are at most 32 factors of $\mathbf{t}$ of length $n$ that are palindrome pairs.

Proof. Let $w$ be an factor of $\mathbf{t}$ of odd length. If $|w| \in\{1,3\}$ the result is clear, so suppose $|w| \geqslant 5$. Blondin-Massé et al. [2] gave the following formula for the number $P_{\mathbf{t}}(n)$ of palindromic factors of length $n$ of $\mathbf{t}$ :

$$
P_{\mathbf{t}}(n)= \begin{cases}1 & n=0 \\ 2 & 1 \leqslant n \leqslant 4 \\ 0 & n \text { odd and } n \geqslant 5, \\ 4 & n \text { even and } 4^{k}+2 \leqslant n \leqslant 3 \cdot 4^{k}, \text { for } k \geqslant 1, \\ 2 & n \text { even and } 3 \cdot 4^{k}+2 \leqslant n \leqslant 3 \cdot 4^{k+1}, \text { for } k \geqslant 1 .\end{cases}
$$

Consequently $w$ is not a palindrome. Suppose $w$ is a product of two palindromes, $u$ and $v$, where $|u|$ is odd. We have two choices for $|u|$, namely $|u| \in\{1,3\}$, and for each choice of length there are two possible values for $u$. Thus there are four choices for $u$. By (1) there are at most four choices for $v$. Since we could have $w=u v$ or $w=v u$, this gives at most 32 possibilities for $w$.

\section{Words with the maximal number of palindrome pair factors}

A word $w$ of length $n$ is called rich (i.e., "rich in palindromes") if it contains the maximum number (viz. $n+1$ ) of distinct palindromic factors [7]. Rich words have been extensively studied. Here we investigate the analogue of richness for palindrome pairs; i.e., we study words of length $n$ that have the maximum possible number of distinct factors that are palindrome pairs.

As it turns out, we are unable to prove anything concerning this problem; we therefore will just present some conjectures based on computer calculations. Computer experiments suggest that for $n \geqslant 1$ the maximum possible number of distinct palindrome pair factors in a binary word of length $n$ is

$$
\left\lceil\frac{n^{2}+2 n+3}{3}\right\rceil
$$


Furthermore, computer experiments also suggest that if $x$ is a binary word of length $n$ beginning with 0 that contains the maximum number of distinct palindrome pair factors, then

- if $n \equiv 0(\bmod 3)$, then $x$ is one of

$$
\begin{array}{lll}
0^{n / 3+1} 1^{n / 3} 1^{n / 3-1}, & 0^{n / 3} 1^{n / 3} 1^{n / 3}, & 0^{n / 3} 1^{n / 3+1} 1^{n / 3-1}, \\
0^{n / 3-1} 1^{n / 3} 1^{n / 3+1}, & 0^{n / 3-1} 1^{n / 3+1} 1^{n / 3}
\end{array}
$$

- if $n \equiv 1(\bmod 3)$, then $x$ is one of

$$
\begin{array}{lll}
0^{\lceil n / 3\rceil} 1^{\lfloor n / 3\rfloor} 1^{\lfloor n / 3\rfloor}, & 0^{\lceil n / 3\rceil} 1^{\lceil n / 3\rceil} 1^{\lfloor n / 3\rfloor-1}, & 0^{\lfloor n / 3\rfloor} 1^{\lfloor n / 3\rfloor} 1^{\lceil n / 3\rceil}, \\
0^{\lfloor n / 3\rfloor} 1^{\lceil n / 3\rceil} 0^{\lfloor n / 3\rfloor}, & 0^{\lfloor n / 3\rfloor-1} 1^{\lceil n / 3\rceil} 1^{\lfloor n / 3\rfloor} ; &
\end{array}
$$

- if $n \equiv 2(\bmod 3)$, then $x$ is one of

$$
0^{\lceil n / 3\rceil} 1^{\lceil n / 3\rceil} 1^{\lfloor n / 3\rfloor}, \quad 0^{\lfloor n / 3\rfloor} 1^{\lceil n / 3\rceil} 0^{\lceil n / 3\rceil} .
$$

Given how simple these words are, it would be nice to prove that they do indeed contain the maximum number of distinct palindrome pair factors.

\section{Minimal non-palindrome pairs}

In this section we look at words somewhat related to those described in the previous section. A minimal non-palindrome pair is a word that is not a palindrome pair but has the property that each of its proper factors is a palindrome pair. It turns out that we can characterize the minimal non-palindrome pairs over the alphabet $\{0,1\}$. In this section all words considered are over the alphabet $\{0,1\}$.

Given a word $w$, a block of $w$ is an occurrence of a factor of $w$ that consists of one or more repetitions of a single letter and that cannot be extended to either side to create a longer such occurrence. The following lemma is easily verified and may be used without reference in the following results.

Lemma 13. Any word with at most three blocks is a palindrome pair.

A block that is neither the first nor the last block in a word $w$ is called an internal block. A block $b$ of $w$ is a maximum block if all other blocks have length at most the length of $b$. Among all maximum blocks, the internal ones are called internal maximum blocks. We now state and prove a critical lemma.

Lemma 14. A minimal non-palindrome pair with an internal maximum block has at most five blocks. 
Proof. Let $w$ be a minimal non-palindrome pair with an internal maximum block $x$ (of ones, say). Note that $|x| \geqslant 2$. Suppose that $w$ has at least six blocks. Since $x$ is internal and $w$ has at least six blocks we may without loss of generality assume that

$$
z_{0}=0 \ldots 0 \underbrace{1 \ldots 1}_{x} 0 \ldots 01 \ldots 1
$$

is a four block factor of $w$ containing $x$ internally. Since

$$
y=0 \ldots 0 \underbrace{1 \ldots 1}_{x} 0 \ldots 01
$$

is a proper factor of $w$, and hence a palindrome pair, and since $|x| \geqslant 2$, it follows that the blocks of 0 's in $z_{0}$ have equal length. Then, since $0^{-1} y$ is also a palindrome pair it follows that these blocks of 0's have length 1 .

We now consider two cases.

Case 1. Assume that $z_{0}$ is not a suffix of $w$. Then, the factor

$$
z_{1}=0 \underbrace{1 \ldots 1}_{x} 01 \ldots 10
$$

occurs in $w$. Since $w$ has at least six blocks it follows that $z_{1}$ is a proper factor of $w$, and hence a palindrome pair. It then follows that $z_{1}$ is a palindrome. Before we proceed, we show that the other case results in the same occurrence.

Case 2. Assume now that $z_{0}$ is a suffix of $w$. Since $w$ has at least six blocks it then follows that

$$
z_{2}=01 \ldots 10 \underbrace{1 \ldots 1}_{x} 0
$$

is a proper factor of $w$, and thus a palindrome pair, and hence a palindrome.

In either case, we find that $w$ contains a palindromic factor of the form $z_{1}$.

We now claim that all blocks of zeros in $w$ have length 1 . Suppose to the contrary that 00 occurs in $w$. Without loss of generality we may assume that there is an occurrence of 00 that begins prior to the start of $z_{1}$. Let $b_{0}$ be the rightmost such occurrence. Let $u_{0}$ be the proper factor of $w$ that begins with this occurrences of $b_{0}$ and ends with the last block of ones in $z_{1}$. Since $u_{0}$ is a proper factor, and hence a palindrome pair, it follows that $b_{0}{ }^{-1} u_{0}$ is a palindrome. However, we then have that the proper factor $u_{0} 1^{-1}$ of $w$ is not a palindrome pair, a contradiction. Hence 00 does not occur in $w$.

Next, we show that all internal blocks of ones in $w$ are maximum blocks. Suppose that there is a block of ones in $w$ which is not a maximum block. Let $b_{1}$ be an occurrence of this with minimum possible distance to $z_{1}$ in $w$. Without loss of generality we may assume that $b_{1}$ precedes $z_{1}$ in $w$. Then, since $b_{1}$ is internal and has minimum possible distance to $z_{1}$ in $w$, it follows that

$$
0 b_{1} 0 \underbrace{1 \ldots 1}_{|x|} 0
$$


is a proper factor of $w$. This factor is not a palindrome pair, a contradiction. Hence, all internal blocks of ones in $w$ are maximum blocks.

It follows from these two properties (all blocks of zeros in $w$ have length 1 and all internal blocks of ones in $w$ have the same length) that $w$ is a palindrome pair. This is easily verified by considering the initial and final blocks of $w$. Hence, we have a contradiction. This completes the proof.

We now make use of this lemma to prove two results which will allow us to construct the class of minimal non-palindrome pairs. By "extending a block" we mean increasing its length by 1 or more.

Lemma 15. The word resulting from extending an internal maximum block in a minimal non-palindrome pair is itself a minimal non-palindrome pair.

Proof. Clearly, the result will follow on repeated application if we show it holds when extending an internal maximum block by one. Let $w$ be a minimal non-palindrome pair with an internal maximum block $x$. Note $|x| \geqslant 2$. Let $w^{\prime}$ be the word resulting from extending $x$ by one and let $x^{\prime}$ be the extension of $x$ in $w^{\prime}$.

We first show that $w^{\prime}$ is not a palindrome pair. Suppose to the contrary that $w^{\prime}=A^{\prime} B$ for some palindromes $A^{\prime}$ and $B$. Assume without loss of generality that $A^{\prime}$ contains at least half of $x^{\prime}$. We may then write $w=A B$ where $A$ is $A^{\prime}$ with one symbol of $x^{\prime}$ removed. Suppose for contradiction that $x^{\prime}$ is contained in $A^{\prime}$. Since $x$ was a maximum block in $w$ it follows that $x^{\prime}$ is unioccurrent in $w^{\prime}$. Hence $x^{\prime}$ is centred in $A^{\prime}$. Clearly in this case $w$ is also a palindrome pair, a contradiction. We conclude that $x^{\prime}$ is split between $A^{\prime}$ and $B$. It then follows from a similar argument that neither $A^{\prime}$ nor $B$ can be contained in $x^{\prime}$. It now follows from Lemma 14 applied to $w$ and the fact that $A^{\prime}$ and $B$ are palindromes that

$$
w^{\prime}=\underbrace{11 \ldots 10 \ldots 0 \overbrace{11 \ldots 1}^{x^{\prime}} \underbrace{1 \ldots 10 \ldots 01 \ldots 1}_{B}}_{A^{\prime}}
$$

and hence

$$
w=\underbrace{11 \ldots 10 \ldots 0 \overbrace{1 \ldots 1}^{x}}_{A} \underbrace{1 \ldots 10 \ldots 01 \ldots 1}_{B}
$$

where 11 is a prefix of $w^{\prime}$, and hence also of $w$. Note that since $w$ is not a palindrome pair, the blocks of zeros in $A$ and $B$ cannot be equal. Let $u$ be the prefix of $w$ ending with the final zero of $B$. Since $w$ has prefix 11 , it follows that $u$ and $1^{-1} u$ are not both palindrome pairs. Since these are proper factors of $w$, we have a contradiction. This completes the proof that $w^{\prime}$ is not a palindrome pair.

We must now prove the minimality of $w^{\prime}$. Suppose for contradiction that $w^{\prime}$ has a proper factor $u^{\prime}$ which is not a palindrome pair. Let $u$ be the corresponding factor in $w$ (note that $u^{\prime}=u$ unless $u^{\prime}$ contains $x^{\prime}$ ). Since $u$ is a proper factor of $w$, it is a palindrome pair. Write $u=a b$ for palindromes $a$ and $b$. Since $u^{\prime}$ is not a palindrome pair it follows 
that $x$ must be contained in $u$, but cannot be centred in either $a$ or $b$. Without loss of generality assume that at least half of $x$ is in $a$. We now consider two cases.

Case 1. Suppose that $x$ is contained in $a$. Without loss of generality we then have by Lemmas 13 and 14 that either

$$
u=\underbrace{1 \ldots 10 \ldots 0 \overbrace{1 \ldots 1}^{x}}_{a} \underbrace{0 \ldots 0}_{b}
$$

or

$$
u=\underbrace{0 \ldots 01 \ldots 10 \ldots 0 \overbrace{1 \ldots 1}^{x} 0 \ldots 0}_{a} \underbrace{0 \ldots 0}_{b}
$$

First, assume the former. Since $1^{-1} u$ is a proper factor of $w$, and hence a palindrome pair, it follows that the blocks of zeros in $a$ and $b$ are equal. This contradicts the assumption that $u^{\prime}$ is not a palindrome pair.

In the latter case, Lemma 14 implies that $w$ consists of 5 blocks. Therefore, $w$ can be obtained by adding some number of zeros to the beginning and end of $u$. However, adding such zeros to the ends of $u$ preserves the property of being a palindrome pair. Consequently, $w$ is a palindrome pair, a contradiction. This completes the case.

Case 2. Suppose that $x$ is split between $a$ and $b$. Since $u^{\prime}$ is not a palindrome pair and by Lemma 14 we may write

$$
u=\underbrace{11 \ldots 10 \ldots 01 \ldots 1}_{a} \underbrace{x}_{b} \underbrace{1 \ldots 10 \ldots}_{a 10 \ldots 01 \ldots 1}
$$

where the blocks of zeros in $a$ and $b$ are not equal. Let $y$ be the prefix of $u$ ending with the last zero of $b$. Note that $y$ and $1^{-1} y$ cannot both be palindrome pairs, but are both proper factors of $w$. This is a contradiction, which completes the proof.

Lemma 16. The word resulting from deleting one digit from an internal maximum block of a minimal non-palindrome pair of length at least 7 is itself either a minimal nonpalindrome pair or is one of $01^{n} 01^{n} 0$ or $10^{n} 10^{n} 1$ for some integer $n \geqslant 1$.

Proof. Let $w^{\prime}$ be a minimal non-palindrome pair of length at least 7 with an internal maximum block $x^{\prime}$ (of ones, say). Note $\left|x^{\prime}\right| \geqslant 2$. Let $w$ and $x$ be obtained from $w^{\prime}$ and $x^{\prime}$ respectively by deleting one digit from $x^{\prime}$. If $w$ is of the form $01^{n} 01^{n} 0$ we are done, so suppose this is not the case.

We first prove that $w$ is not a palindrome pair. Suppose to the contrary and write $w=A B$ for palindromes $A$ and $B$. Without loss of generality assume that at least half of $x$ is in $A$. Since $w^{\prime}$ is not a palindrome pair it follows that $x$ is not centred in either $A$ or $B$, nor is either $A$ or $B$ contained in $x$. There are two cases to now consider.

Case 1. Assume that $x$ is contained in $A$. By Lemmas 13 and 14 there are without loss of generality two further possibilities. Either

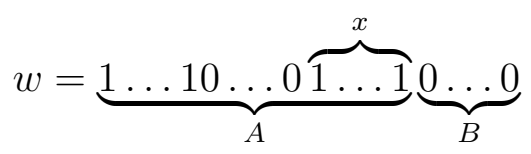


or

$$
w=\underbrace{0 \ldots 01 \ldots 10 \ldots 0 \overbrace{1 \ldots 1}^{x} 0 \ldots 0}_{A} \underbrace{0 \ldots 0}_{B}
$$

First, assume the former. Since $w^{\prime}$ is not a palindrome pair it follows that the blocks of zeros in $w^{\prime}$ are not equal. Since $1^{-1} w^{\prime}$ is a proper factor of $w^{\prime}$, and hence a palindrome pair, it follow that $w^{\prime}=10 \ldots 110 \ldots 0$. Since $x^{\prime}$ is maximum in $w^{\prime}$ it then follows that $\left|w^{\prime}\right|=6$, a contradiction.

Next, assume the latter. Note that since $w^{\prime}$ is not a palindrome pair, the blocks of ones in $w^{\prime}$ have different lengths. Let $v$ be the suffix of $w^{\prime}$ starting with the first one of $w^{\prime}$. Since $v$ is a proper factor of $w^{\prime}$, and hence a palindrome pair, it follows that the blocks of zeros in $v$ are equal. Applying a similar argument to the first four blocks of $w^{\prime}$ we get that the first two blocks of zeros in $w^{\prime}$ are also equal. Since $A$ is a palindrome it then follows that $B=\varepsilon$. Since $0^{-1} w^{\prime}$ is a palindrome pair it follows that the blocks of zeros in $w^{\prime}$ have length 1 . Thus $w^{\prime}=01^{\left|x^{\prime}\right|-1} 01^{\left|x^{\prime}\right|} 0$, and hence $w=01^{\left|x^{\prime}\right|-1} 01^{\left|x^{\prime}\right|-1} 0$, which is a contradiction. This completes the case.

Case 2. Assume $x$ is split between $A$ and $B$. By Lemma 14 it follows that

$$
w=\underbrace{1 \ldots 10 \ldots 0 \overbrace{1 \ldots 1}^{x}}_{A} \underbrace{1 \ldots 10 \ldots 01 \ldots 1}_{B}
$$

Since $w^{\prime}$ is not a palindrome pair it follows that the blocks of zeros in $w^{\prime}$ are not equal. Thus, the proper factor of $w^{\prime}$ obtained from $w^{\prime}$ by deleting its final block of ones is not a palindrome pair. This contradiction completes the proof that $w$ is not a palindrome pair.

It remains to prove that $w$ is minimal. Suppose that some proper factor $u$ of $w$ is not a palindrome pair. Let $u^{\prime}$ be the corresponding proper factor of $w^{\prime}$. Since $u^{\prime}$ is a palindrome pair, while $u$ is not, $u$ must contain $x$. Write $u^{\prime}=a b$ for palindromes $a$ and $b$. Without loss of generality we may assume that at least half of $x^{\prime}$ is in $a$. Clearly, $x^{\prime}$ is not centred in either $a$ or $b$. By Lemma 14 without loss of generality we again consider two cases.

Case 1. Assume that $x^{\prime}$ is contained in $a$. Then either

$$
u^{\prime}=\underbrace{1 \ldots 10 \ldots 0 \overbrace{1 \ldots 1}^{x^{\prime}}}_{a} \underbrace{0 \ldots 0}_{b}
$$

or

$$
u^{\prime}=\underbrace{0 \ldots 01 \ldots 10 \ldots 0 \overbrace{1 \ldots 1}^{x^{\prime}} 0 \ldots 0}_{a} \underbrace{0 \ldots 0}_{b}
$$

First, assume the former. Since $u$ is not a palindrome pair it follows that the blocks of zeros in $u^{\prime}$ are not equal. Thus $1^{-1} u^{\prime}$ is a proper factor of $w^{\prime}$ which is not a palindrome pair, a contradiction. Next, assume the latter. Lemma 14 implies that $w^{\prime}$ is obtained from $u^{\prime}$ by adding some number of zeros to the beginning and end of $u^{\prime}$. However, this would mean that $w^{\prime}$ is a palindrome pair, which is a contradiction. This contradiction completes the proof. 
One final Lemma is needed for our main result.

Lemma 17. The only minimal non-palindrome pairs which do not have internal maximum blocks are $11(01)^{n} 00$ and $00(10)^{n} 11$ for each $n \geqslant 1$.

Proof. Clearly, these words are minimal non-palindrome pairs. Let $w$ be a minimal nonpalindrome pair with no internal maximum blocks that is not one of the words given in the statement of the lemma. From left to right, let $b_{i}$ be the $i$ th block of ones in $w$. Assume without loss of generality that $b_{0}$ is both a prefix and a maximum block of $w$. We prove that $w$ does not have an internal block of zeros with length at least 2. Suppose to the contrary that such a block exists. Let $u$ be the shortest prefix of $w$ with suffix 00 . Since $u$ is a proper factor of $w$, and hence a palindrome pair, it then follows $u=b_{0} 00$. Since $w$ is not a palindrome pair it follows that $w$ has at least four blocks. Let $v$ be the smallest four block prefix of $w$. We have $v=b_{0} 0 \ldots 0 b_{1} 0$, which is not a palindrome pair. Thus, $v=w$. Now, since $1^{1-\left|b_{0}\right|} v$ is a proper factor of $w$, and hence a palindrome pair, it follows that $\left|b_{1}\right|=1$. Finally, since $1^{-1} v$ is a proper factor of $w$, and hence a palindrome pair, it follows that $b_{0}=11$, and hence since $b_{0}$ is maximum and $w$ is not a palindrome pair, that $w=110100$. We may conclude that $w$ has no internal block of at least two zeros. We now consider two cases.

Case 1. Assume $w$ has at least five blocks. Thus, $y_{0}=b_{0} 0 b_{1} 0 b_{2}$ is a prefix of $w$. Since $w$ is not a palindrome pair it follows that $y_{1}=y_{0} 0$ is also a prefix of $w$. Since $b_{0}{ }^{-1} y_{1}$ is a proper factor of $w$, and hence a palindrome pair, it follows that $\left|b_{1}\right|=\left|b_{2}\right|$. Since $w$ is not a palindrome pair it then follows that $y_{2}=y_{1} b_{3} 0$ is a prefix of $w$. We then argue as before to show that $\left|b_{3}\right|=\left|b_{2}\right|$, and continue until we reach the final block of $w$. Since $w$ is not a palindrome pair, we have that $w=b_{0} 0 b_{1} 0 \ldots b_{k} 0 \ldots 0$ for some integer $k \geqslant 1$ (note that 00 is a suffix of $w$ and $\left|b_{i}\right|=\left|b_{j}\right|$ for any $i, j$ such that $1 \leqslant i<j \leqslant k$ ). If $\left|b_{k}\right|>1$, then the suffix of $w$ starting with the last 1 of $b_{k-1}$ is a proper factor of $w$, but not a palindrome pair, a contradiction. Thus all internal blocks of ones have length 1 . Thus $z=11(01)^{r} 00$ is a factor of $w$ for some $k \geqslant 1$, and hence, as $z$ is not a palindrome pair, $z=w$. This completes the case.

Case 2. Assume now that $w$ has at most four blocks. Thus

$$
w=b_{0} 0 b_{1} 0 \ldots 0
$$

Since $w$ is not a palindrome pair it follows that 00 is a suffix of $w$. Since $10 b_{1} 00$ is a proper factor of $w$, and hence a palindrome pair, it follows that $\left|b_{1}\right|=1$. Thus $w$ contains, and is hence equal to, 110100. This completes the proof.

We now construct the class of minimal non-palindrome pairs inductively. It is easily verified that every word of length five or less is a palindrome pair. The minimal nonpalindrome pairs of length six are given below. For $i \geqslant 7$, to generate the minimal non-palindrome pairs of length $i$ :

- Extend any internal maximum block in a minimal non-palindrome pair of length $i-1$ by one. 
- If $i$ is even, add the words $11(01)^{\frac{i-4}{2}} 00,01^{\frac{i-4}{2}} 01^{\frac{i-4}{2}+1} 0,10^{\frac{i-4}{2}} 10^{\frac{i-4}{2}+1} 1,01^{\frac{i-2}{2}} 0^{\frac{i-2}{2}} 1$ and their reverses.

The minimal non-palindrome pairs for the first few $i$ are given in the following table. For conciseness, reverses and words with prefix 1 have been omitted.

\begin{tabular}{|c||c|c|c|c|c|}
\hline $\mathrm{i}$ & 6 & 7 & 8 & 9 & 10 \\
\hline & 001011 & 0011101 & 00101011 & 001111101 & 0010101011 \\
& 001101 & 0100011 & 00111101 & 010000011 & 0011111101 \\
& 010011 & 0101110 & 01000011 & 010111110 & 0100000011 \\
& 010110 & 0110001 & 01011110 & 011000001 & 0101111110 \\
& 011001 & 0111001 & 01100001 & 011011110 & 0110000001 \\
& & & 01101110 & 011100001 & 0110111110 \\
& & & 01110001 & 011110001 & 0111000001 \\
& & & 01111001 & 011111001 & 0111011110 \\
& & & & & 0111100001 \\
& & & & & 0111110001 \\
& & & & & 0111111001 \\
\hline
\end{tabular}

Table 1: List of short minimal non-palindrome pairs

This leads us to our main result.

Theorem 18. The minimal non-palindrome pairs are exactly those words described above.

Proof. It is easily verified that those words of length six given in the table, as well as those described in part two of the construction above are minimal non-palindrome pairs. It then follows from Lemma 15 that all the constructed words are also minimal non-palindrome pairs.

To show that these are the only minimal non-palindrome pairs, let $w^{\prime}$ be a minimal non-palindrome pair of length $i \geqslant 7$. If $w^{\prime}$ has no internal maximum blocks, then by Lemma $17, w^{\prime}$ is either $11(01)^{\frac{i-4}{2}} 00$ or its reverse, and is therefore accounted for by our construction.

If $w^{\prime}$ has exactly one internal maximum block, then $w^{\prime}$ can be obtained by extending an internal maximum block in some word $w$ of length $i-1$. By Lemma $16 w$ is either a minimal non-palindrome pair, or is one of $01^{\frac{i-4}{2}} 01^{\frac{i-4}{2}} 0$ or $10^{\frac{i-4}{2}} 10^{\frac{i-4}{2}} 1$. Again $w^{\prime}$ is accounted for by our construction.

If $w^{\prime}$ has more than one internal maximum block, then without loss of generality $w^{\prime}$ contains a factor $01^{k} 0^{k} 1$ for some $k \geqslant 2$. However this factor is not a palindrome pair. It follows that this factor is not a proper factor and hence that $w^{\prime}=01^{\frac{i-2}{2}} 0^{\frac{i-2}{2}} 1$. Again $w^{\prime}$ is accounted for by our construction. This concludes the proof.

Corollary 19. Let npp $(i)$ be the number of minimal non-palindrome pairs of length $i$. Then $n p p(i)=0$ if $i<6$ and for all $j \geqslant 3$,

$$
n p p(2 j)=n p p(2 j+1)=8 j-12 .
$$


Proof. One first checks that there are 12 minimal non-palindrome pairs of length 6 . Let $i>6$ be odd. Every minimal non-palindrome pair of length $i-1$ with exactly 1 internal maximum block produces one of length $i$ by extending this internal maximum block. The word $11(01)^{\frac{i-5}{2}} 00$ and its reversal produce no minimal non-palindrome pairs of length $i$, since they have no internal maximum blocks to extend. The word $01^{\frac{i-3}{2}} 0^{\frac{i-3}{2}} 1$ and its reversal each produce two minimal non-palindrome pairs, since they each have two internal maximum blocks. Thus there is no net increase in the number of minimal non-palindrome pairs when going from length $i-1$ to $i$, and so we have $n p p(i)=n p p(i-1)$.

On the other hand, if $i$ is even, then every minimal non-palindrome pair of length $i-1$ produces one minimal non-palindrome pair of length $i$. Additionally, our construction adds eight new words of length $i$. Thus there are

$$
\begin{aligned}
& \operatorname{npp}(i-1)+8 \\
= & \operatorname{npp}(i-2)+8 \quad(\text { since } i-1 \text { is odd) } \\
= & 4(i-2)-12+8 \quad \text { (inductively) } \\
= & 4 i-12
\end{aligned}
$$

minimal non-palindrome pairs of length $i$.

Writing $i=2 j$ if $i$ is even and $i=2 j+1$ if $i$ is odd, the above gives $n p p(2 j)=$ $n p p(2 j+1)=8 j-12$, as required.

\section{Conclusion}

Here we mention some interesting open questions raised by the previous results. The first one is an obvious one, although we shall see shortly that the answer may, in fact, be "no".

Question. Does property PP characterize some interesting class of words?

We can also define a complexity function based on palindrome pairs. Recall that the factor complexity function $C_{\mathbf{w}}(n)$ counts the number of factors of $\mathbf{w}$ of length $n$. Similarly, the palindrome complexity function $P_{\mathbf{w}}(n)$ counts the number of palindromic factors of $\mathbf{w}$ of length $n$. We could therefore define a palindrome pair complexity function $\operatorname{PP}_{\mathbf{w}}(n)$ that counts the number of factors of $\mathbf{w}$ of length $n$ that are palindrome pairs. Property PP could then be rephrased as " $C_{\mathbf{w}}(n)=P P_{\mathbf{w}}(n)$ for infinitely many $n$."

Problem. Explore the relationships between the functions $C_{\mathbf{w}}(n), P_{\mathbf{w}}(n)$, and $P P_{\mathbf{w}}(n)$.

It is known that if $\mathbf{w}$ has linear factor complexity then its palindromic complexity is bounded [1]. We have already seen above that this is not true for the palindrome pair complexity. The results of Section 3 give upper and lower bounds for the palindrome pair complexity of the Thue-Morse word for certain values of $n$.

Problem. Give explicit formulas for the palindrome pair complexity of words such as the Fibonacci word and the Thue-Morse word. 
Further to the question posed above regarding whether property $\mathbf{P P}$ characterizes some interesting class of words, one might wonder if, for instance, property $\mathbf{P P}$ implies $O(n)$ factor complexity. Unfortunately, this is not the case. Suppose we perform the construction of the word $\mathbf{p}$ in Proposition 9 by defining the $w_{i}$ 's as follows: $w_{i}=B_{i} B_{i}^{R}$, where $B_{i}$ is a de Bruijn sequence of order $\left|p_{i}\right|+1$; that is, $B_{i}$ contains every binary word of length $\left|p_{i}\right|+1$ exactly once (see, for instance, [5]). Then $\mathbf{p}$ contains at least $2^{\left|p_{i}\right|+1}$ factors of length $\left(\left|p_{i}\right|+1\right)^{2}$. If we set $n_{i}=\left(\left|p_{i}\right|+1\right)^{2}$, then we see that $C_{\mathbf{p}}\left(n_{i}\right) \geqslant 2^{\sqrt{n_{i}}}$. So there are words that are not so "nice" that still have property $\mathbf{P P}$.

The following would show that property $\mathbf{P P}$ is a property of Sturmian words that does not carry over to episturmian words. In principle, it could be resolved using the Walnut Prover, as described in the proof of Theorem $10^{1}$.

Conjecture 20. The Tribonacci word does not have property PP.

In Section 4 we already stated the problem of characterizing the binary words of each length that have the maximum possible number of distinct palindrome pair factors. Of course, the most interesting open problem is to resolve the Frid-Puzynina-Zamboni Conjecture.

\section{Acknowledgments}

We would like to thank Luke Schaeffer for his help with using the Walnut Prover.

\section{References}

[1] J.-P. Allouche, M. Baake, J. Cassaigne, D. Damanik. Palindrome complexity. Theoret. Comput. Sci. 292:9-31, 2003.

[2] A. Blondin-Massé, S. Brlek, A. Garon, S. Labbé. Combinatorial properties of $f$ palindromes in the Thue-Morse sequence. Pure Math. Appl. (PU.M.A.) 19:39-52, 2008.

[3] A. de Luca, F. Mignosi. Some combinatorial properties of Sturmian words. Theoret. Comput. Sci. 136:361-385, 1994.

[4] S. Fischler. Palindromic prefixes and episturmian words, J. Combin. Theory Ser. A 113:1285-1286, 2006.

[5] H. Fredricksen, J. Maiorana. Necklaces of beads in $k$ colors and $k$-ary de Bruijn sequences. Discrete Math. 23:207-201, 1978.

[6] A. E. Frid, S. Puzynina, L. Q. Zamboni. On palindromic factorization of words. Adv. in Appl. Math. 50:737-748, 2013.

\footnotetext{
${ }^{1}$ When the second author attempted to perform the necessary calculations using the Walnut Prover, his computer ran out of memory. Apparently the current version of Walnut does not handle Tribonacci-base computations efficiently.
} 
[7] A. Glen, J. Justin, S. Widmer, L. Q. Zamboni. Palindromic richness. European J. Combin. 30:510-531, 2009.

[8] D. Goč, D. Henshall, J. Shallit. Automatic theorem-proving in combinatorics on words. Int. J. Found. Comput. Sci. 24:781-798, 2013.

[9] C. Guo, J. Shallit, A. Shur. On the combinatorics of palindromes and antipalindromes. Preprint available at arXiv: 1503.09112.

[10] S. Holub, M. Müller. Binary words with the fewest unbordered conjugates. Preprint available at arXiv: 1504.02222.

[11] R. Kemp. On the number of words in the language $\left\{w \in \Sigma^{*} \mid w=w^{R}\right\}^{2}$. Discrete Math. 40:225-234, 1982.

[12] M. Lothaire. Algebraic Combinatorics on Words. Cambridge, 2002.

[13] G. Melançon. Lyndon words and singular factors of sturmian [sic] words. Theoret. Comput. Sci. 218:41-59, 1999.

[14] L. Schaeffer, J. Shallit. Closed, rich, privileged, trapezoidal, and balanced words in automatic sequences. Preprint available at arXiv:1508.02074. 